\title{
ADEPT Sounding Rocket One (SR-1) Flight Experiment Overview \\ Paul Wercinski
}

NASA Ames Research Center

Moffett Field, CA

Co-Authored by B. Smith, B. Yount, A. Cassell, C. Kruger,

C. Brivkalns, A. Makino, S. Dutta, S. Ghassemieh, S. Wu,

S. Battazzo, O. Nishioka, E. Venkatapathy

2017 IEEE Aerospace Conference

Big Sky, MT

Track 2: Space Missions, Systems, and Architectures

Session 2.03 Systems and Technologies for Landing on Planets, the Moon, Earth and Small Bodies 


\section{What is this talk about?}

My goal is for you to walk away with an understanding of the ADEPT technology, overview of the SR-1 flight experiment, SR-1 system description and capabilities, development test summary, and longer term mission infusion

Presentation Outline

- ADEPT Technology overview

- SR-1 Flight Experiment Con-Ops

- Flight Experiment Success Criteria

- SR-1 Subsystem Description

- Risk-based Development Testing Approach

- ADEPT technology infusion options

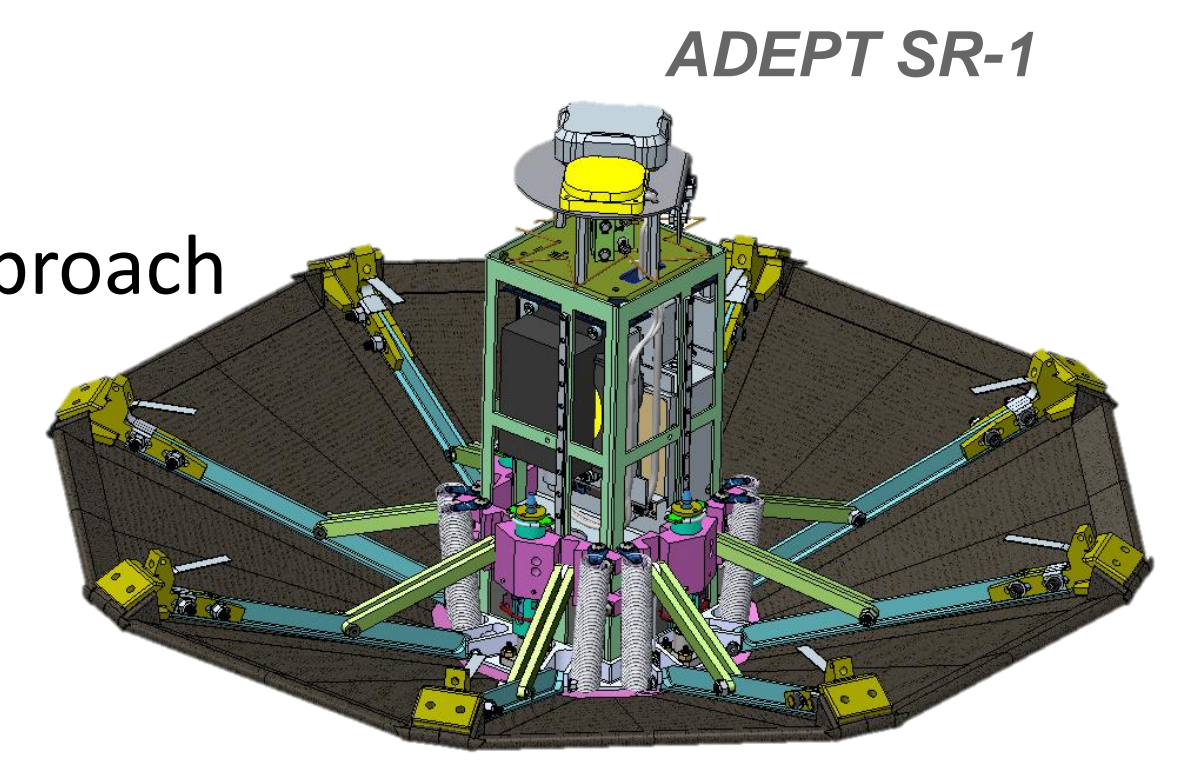




\section{Adaptable Deployable Entry and Placement Technology (ADEPT)}

ADEPT is a novel Entry, Descent, and Landing (EDL) architecture enabled with multi-layer, flexible woven carbon fabric

- Stowed at launch and deploys prior to atmosphere entry

- Serves as both heat shield and primary structure

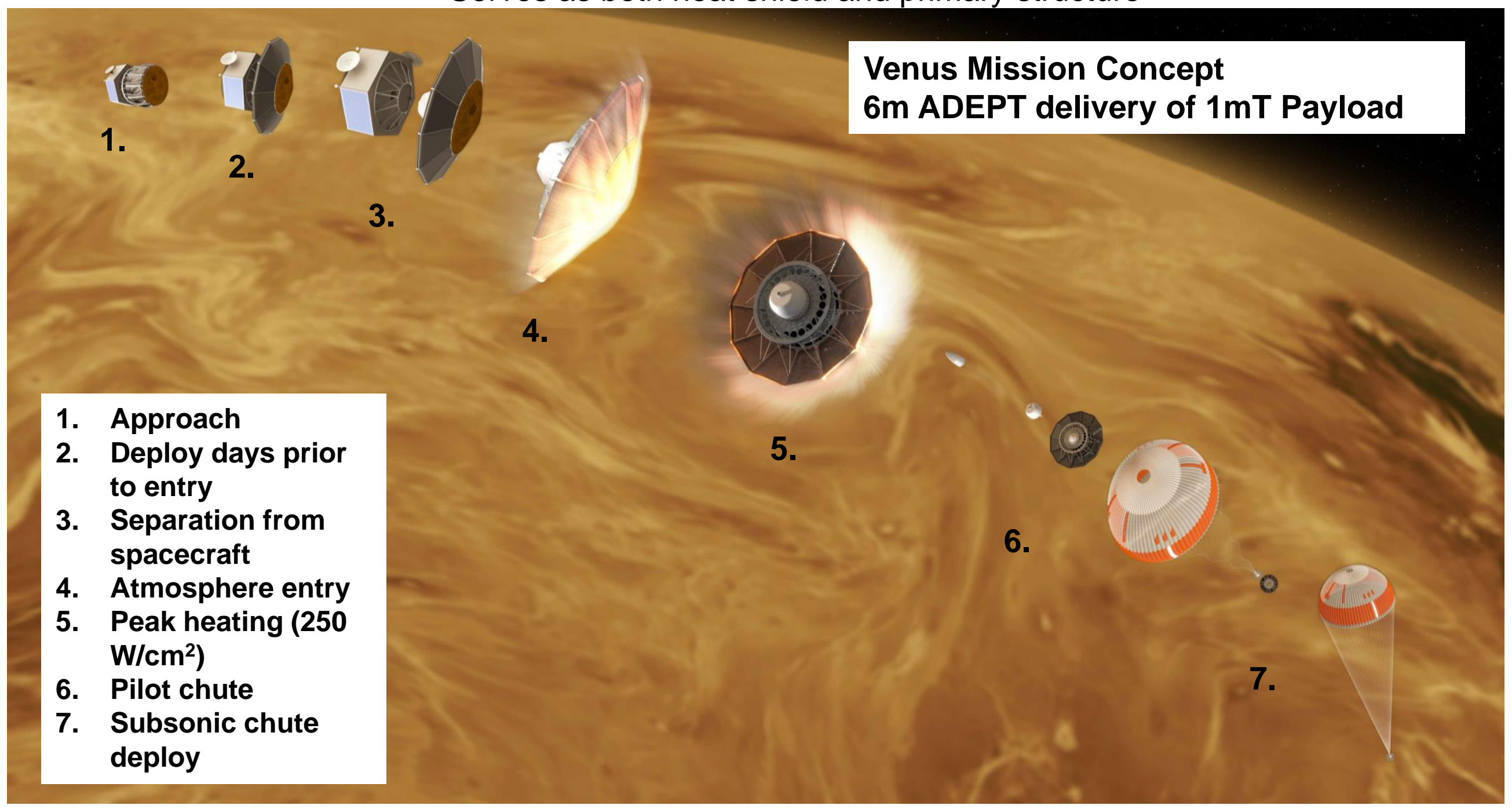




\section{Technology Maturation Strategy}

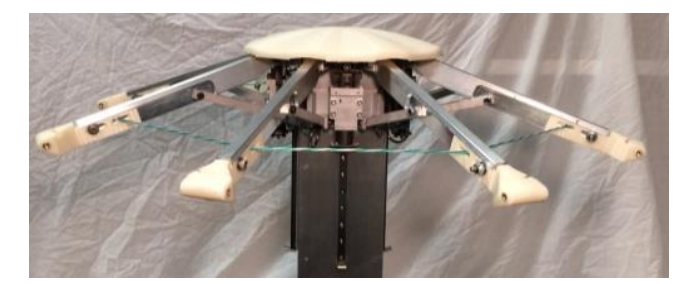

Deployment Prototype Demonstrator (FY15-16)

SR-1 Sounding Rocket Flight Test (FY17-18)
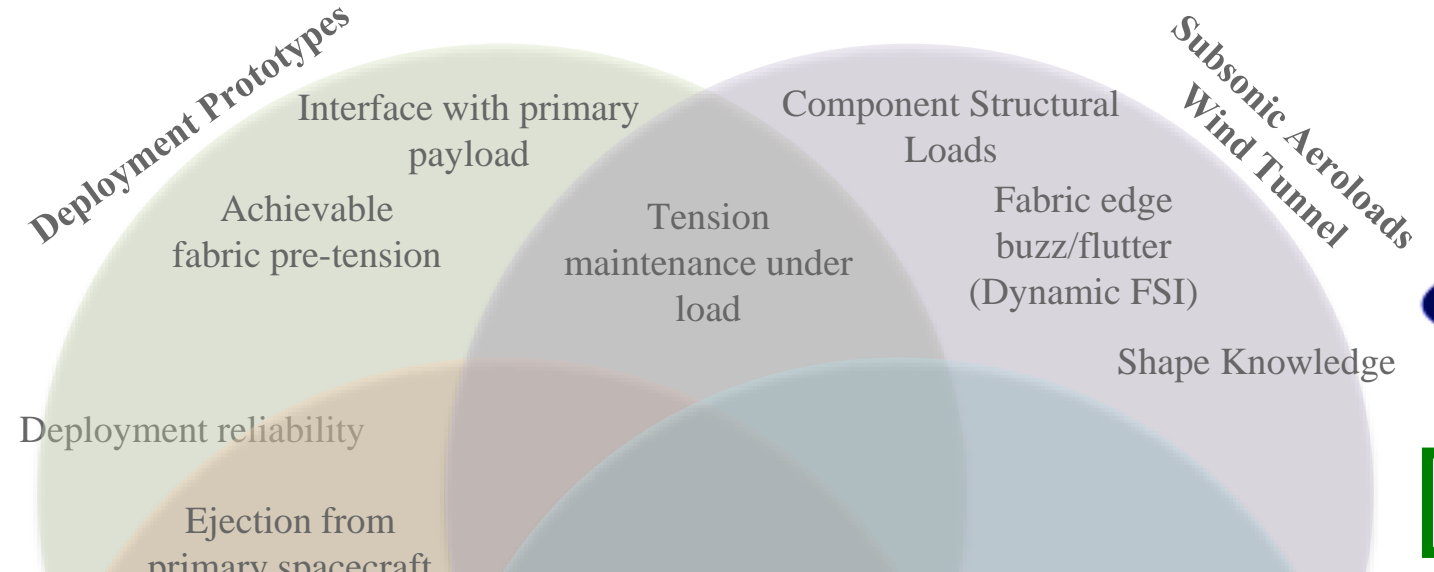

primary spacecraft

Tip-off rates

Fabric System Stowage

Pre-Entry orientation

Supersonic Aerostability

So Exo-atmospheric Deploy

N

Fabric system

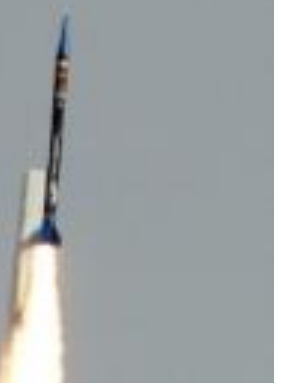

Shape Knowledge

Tech Maturation for Mission Infusion

FSI

Thermo-structural loads

Gore Deflection

(Static FSI)

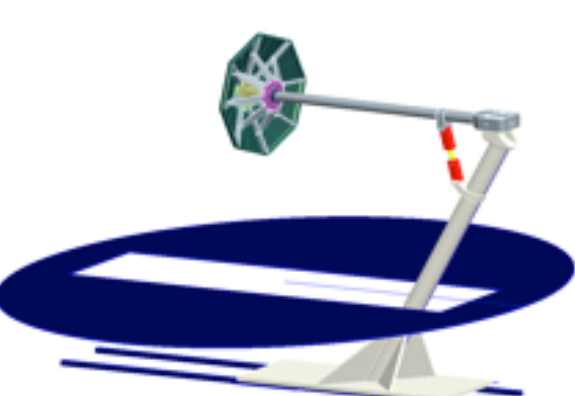

$7 \times 10$ Wind-tunnel Aeroloads test (FY15)

SPRITE C

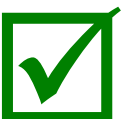
System level Arcjet testing (FY15)

- GCD approved (Aug 2016) SR-1 Sounding Rocket Flight Experiment

- Demonstrating exo-atmospheric deployment and supersonic stability

- Aggressive schedule -> 1 year between PDR and Launch!

- Launch in late CY 2017 


\section{SR-1 Flight Experiment Overview}

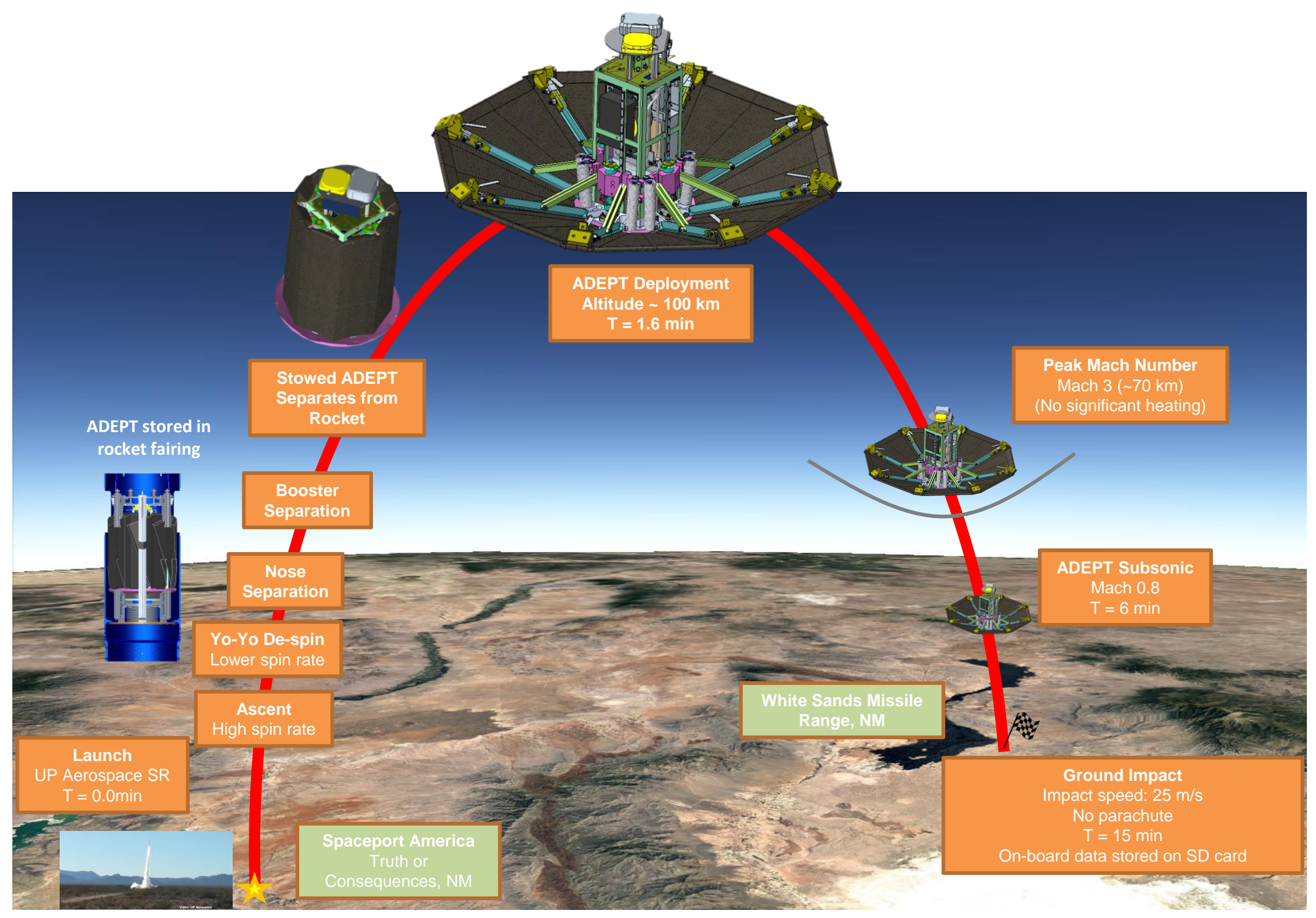




\section{SR-1 Animation movie}




\section{ADEPT SR-1 Flight Experiment Key Performance Parameters and Success Criteria}

KPP-SR1-1: Exo-atmospheric deployment to an entry configuration

Project Goal: Full, locked deployment before reaching $80 \mathrm{~km}$ altitude on descent, to $70^{\circ}$ forebody cone angle

KPP-SR1-2: Aerodynamic stability without active control

Project Goal: Does not tumble before ground impact;

\section{SR-1 Flight Test Success Criteria}

A. ADEPT separates from the sounding rocket prior to apogee

B. ADEPT does not re-contact any part of the launch vehicle after separation

C. ADEPT reaches an apogee greater than $100 \mathrm{~km}$.

D. ADEPT achieves fully deployed and locked configuration prior to reaching $80 \mathrm{~km}$ altitude on descent

E. Obtain video of deployed ADEPT to observe fabric response and flight dynamics during entry

F. Obtain data necessary to reconstruct ADEPT 6 DOF descent trajectory

- Data Sources to Verify Success Criteria

On-board data (Avionics data and GoPro camera) stored for post-launch recovery White Sands Missile Range (WSMR) ground tracking data 


\section{SR-1 Layout and Subsystems}

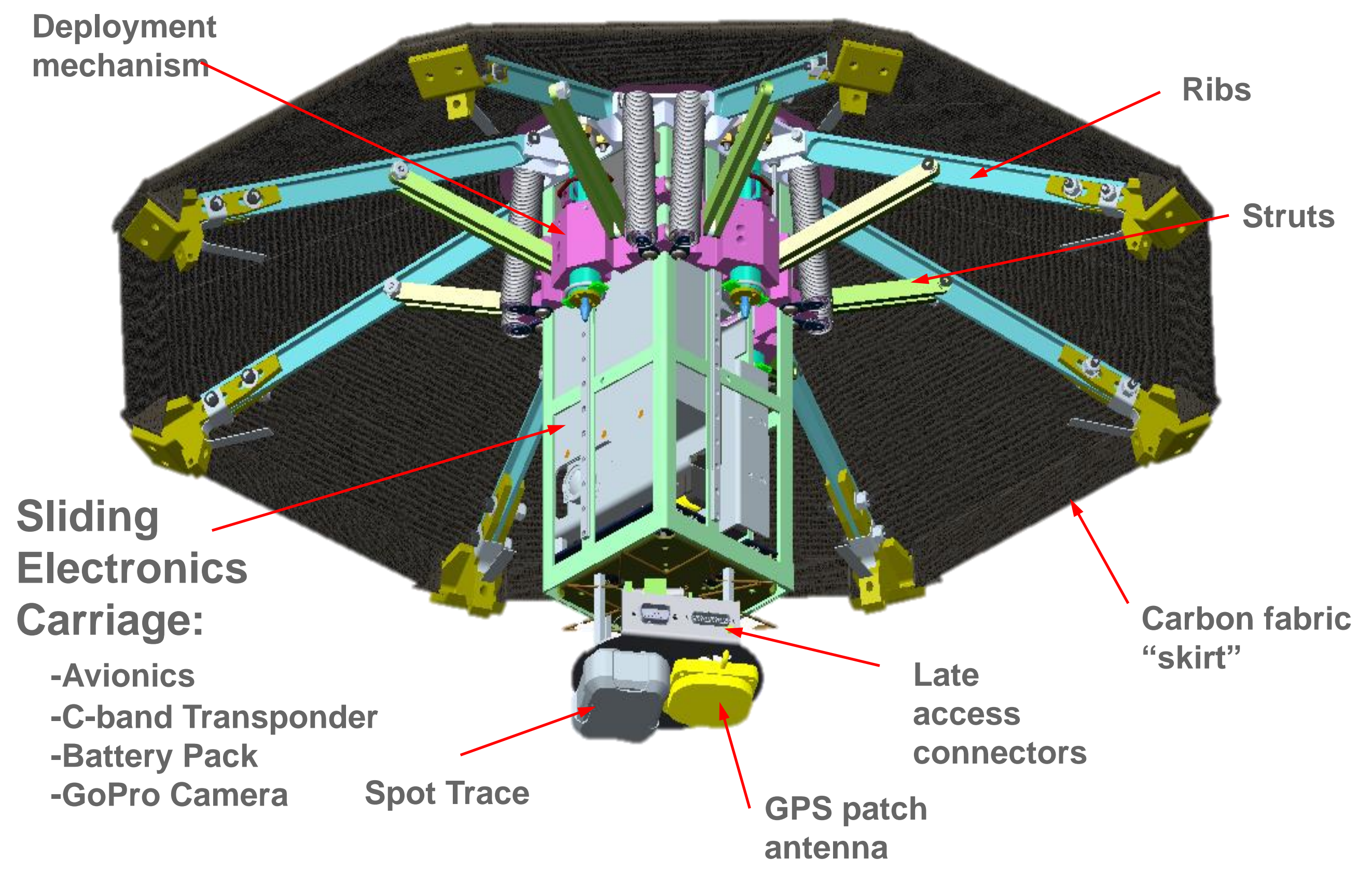




\section{SR-1 Electronics Carriage}

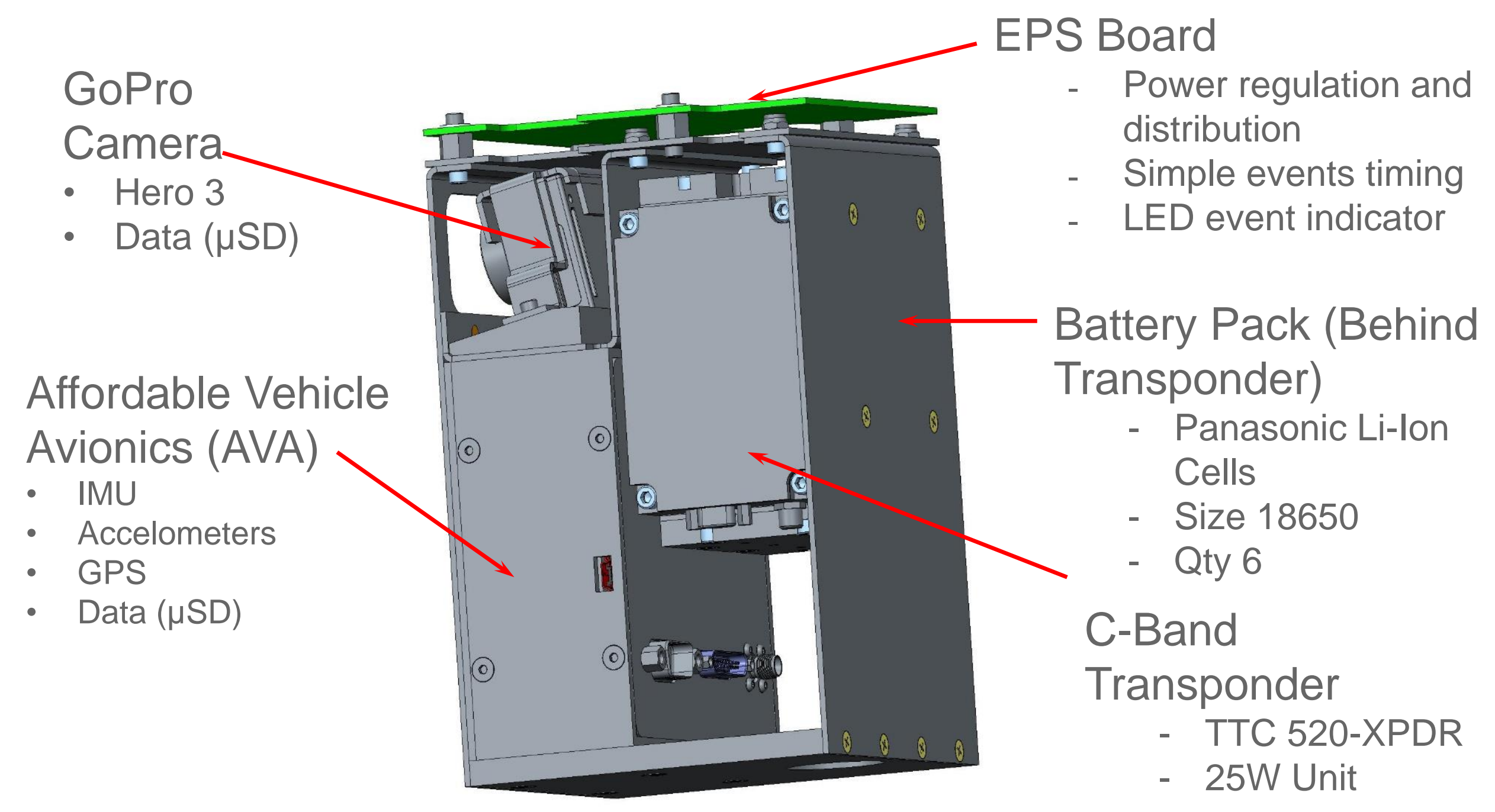

\section{Electonics Carriage \\ Supports on-board data collection and storage Supports Ground Tracking facilities}




\section{How SR-1 Data Sources will be Used}

GoPro Camera

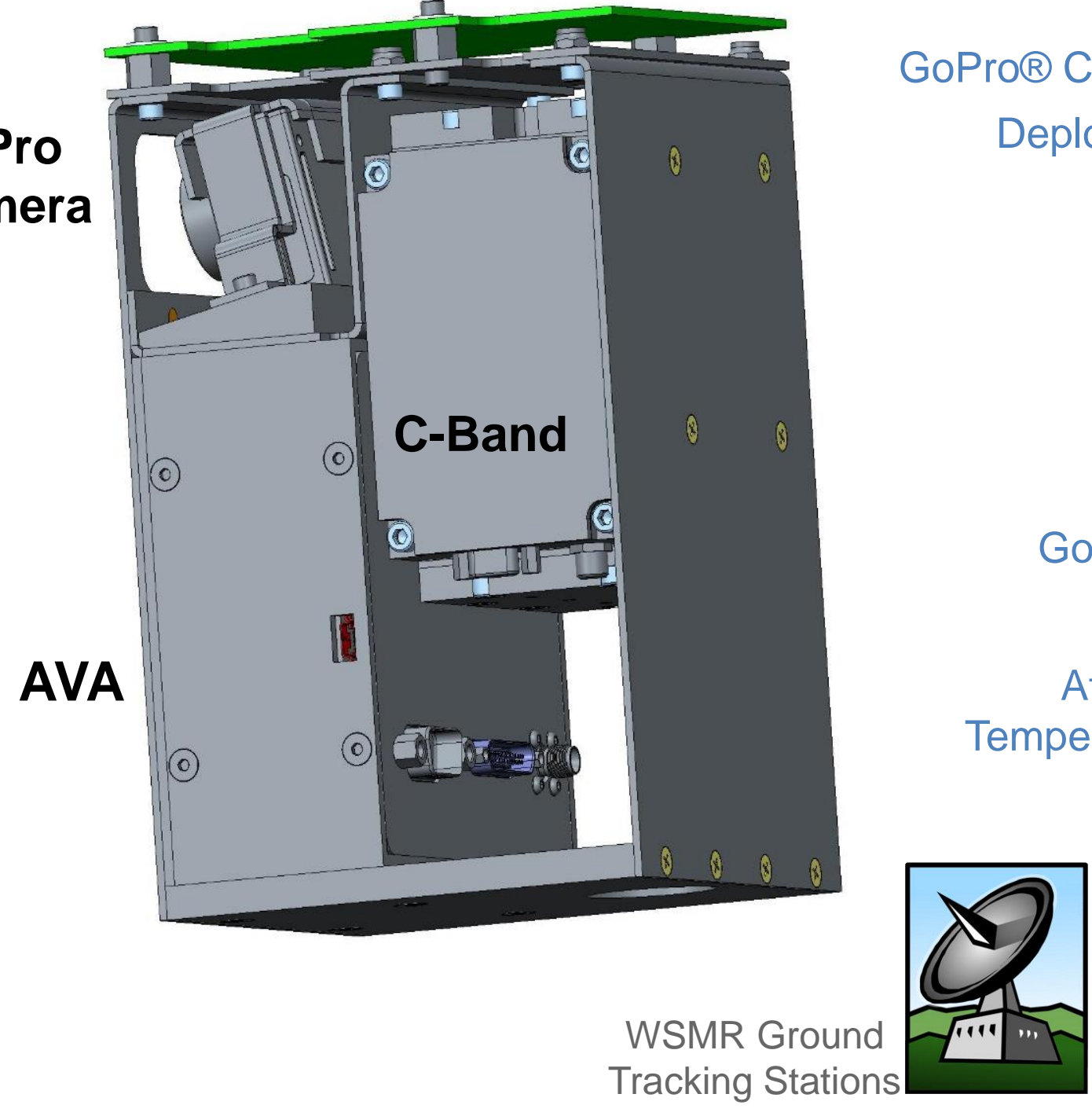

WSMR Ground Tracking Stations
GoPro® Camera on Launch Vehicle

Deployment Confirmation LED

USE: Confirm full and locked deployment

\section{Electonics Carriage}

SD cards must survive $\sim 25 \mathrm{~m} / \mathrm{s}$ ( $54 \mathrm{mph}$ ) impact velocity! 


\section{Shock Testing of SD Cards}

\section{(Drop Testing to Assess Impact Survival)}
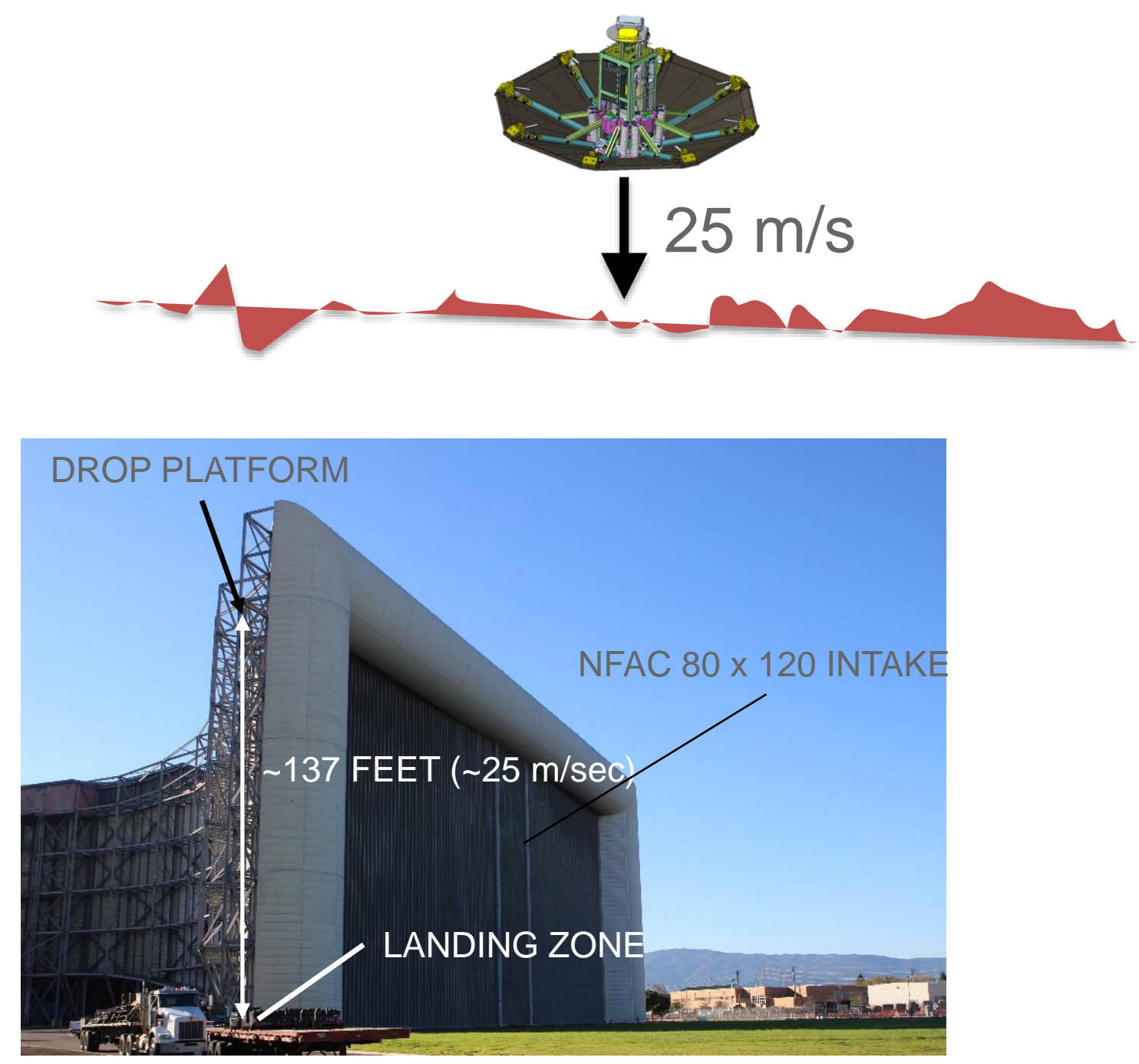

- Drop test location allows matching of flight article impact velocity

- Drop test configuration allows controlled impact testing of impact attenuator and SR-1 electronics carriage

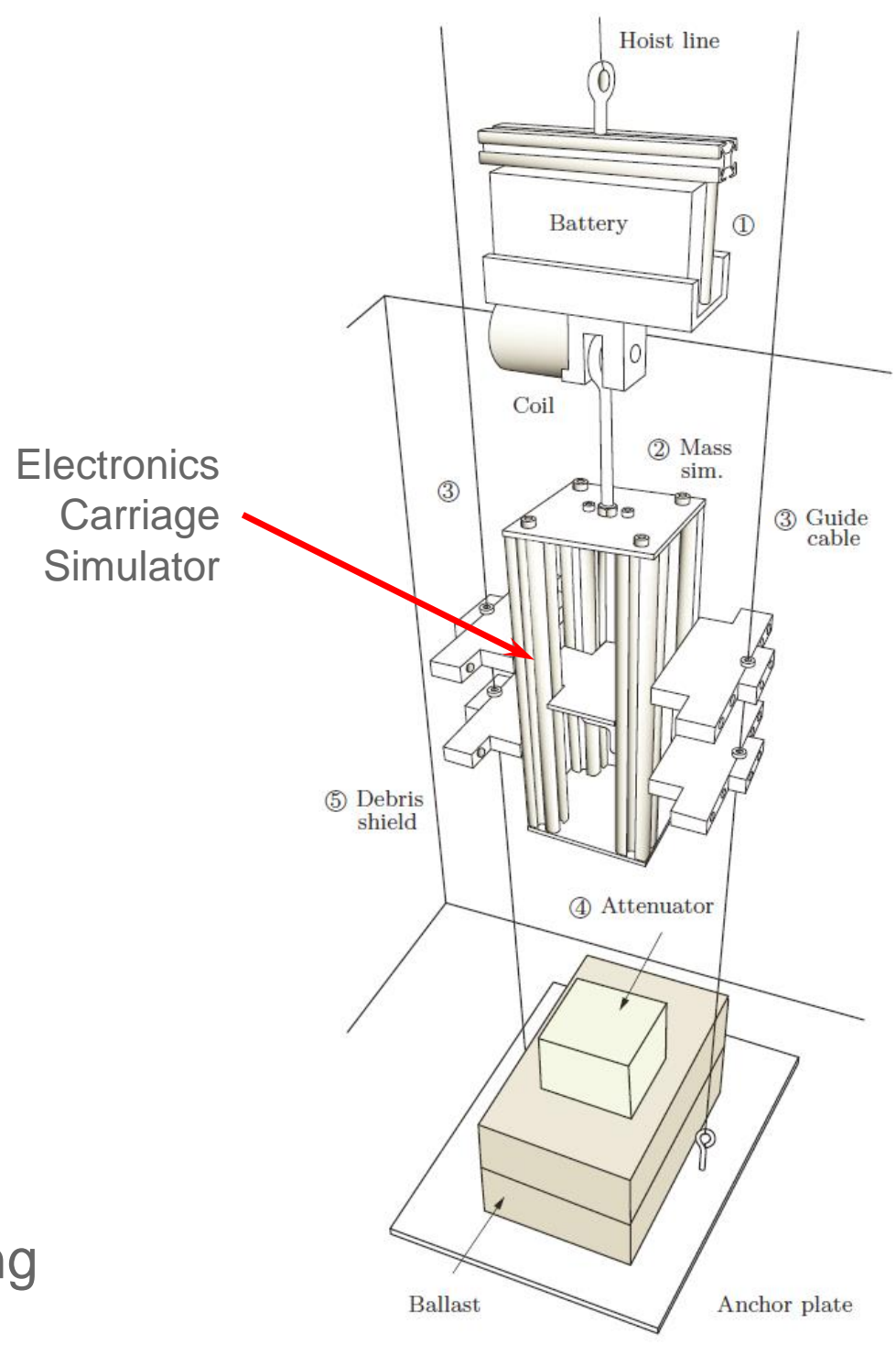




\section{Shock Testing of SD Cards}

\section{(Drop Testing to Assess Impact Survival)}




\section{Summary}

- ADEPT SR-1

- "First step" Flight experiment demonstrating ADEPT

- Looking beyond SR-1...

- Small spacecraft by using an ADEPT EDL system to overcome volume limits

- Secondary payloads to Venus, Mars, and LEO entry are feasible near-term applications

- Nano-ADEPT provides technology development extensible to large ADEPT applications

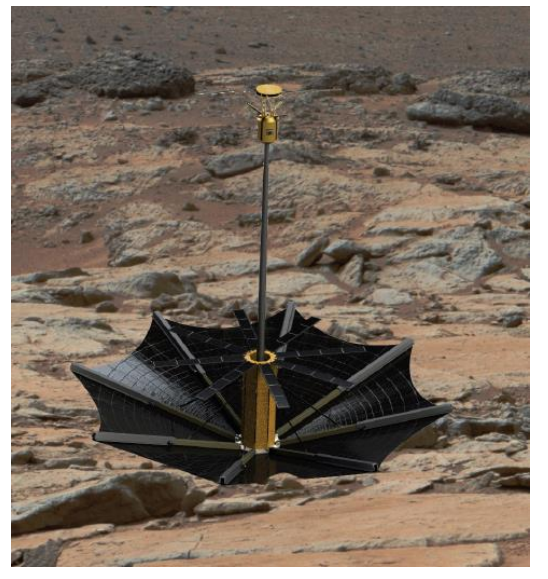

$1 \mathrm{~m}$ ADEPT Mars Lander Malin SSS Concept (2014)

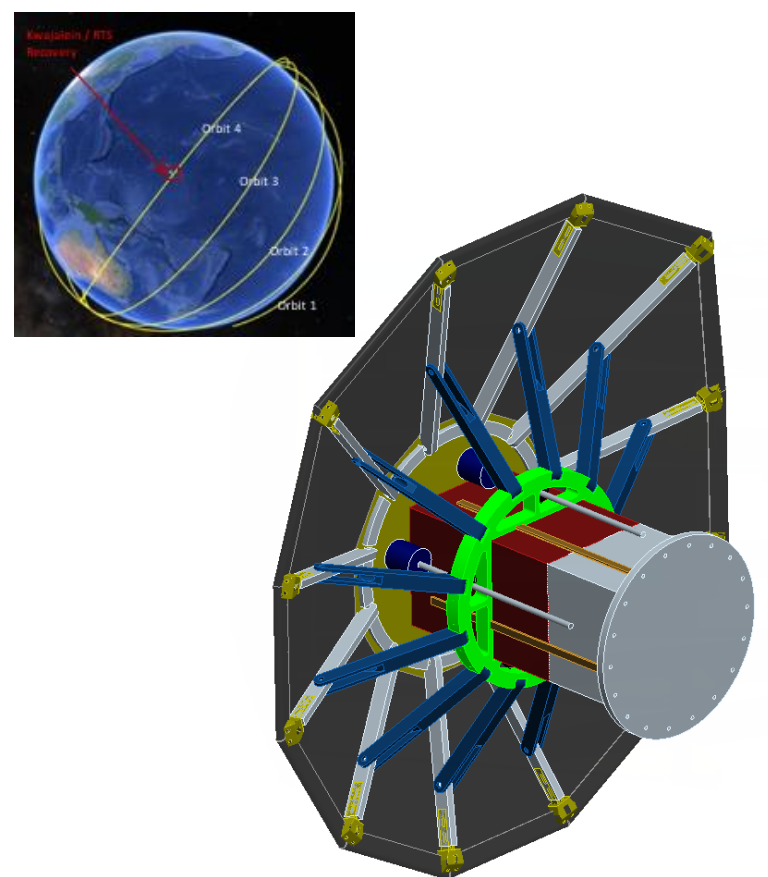

1.5m Lifting ADEPT LEO Flight Test Concept NASA Ames \& JHU-APL Study (2016)

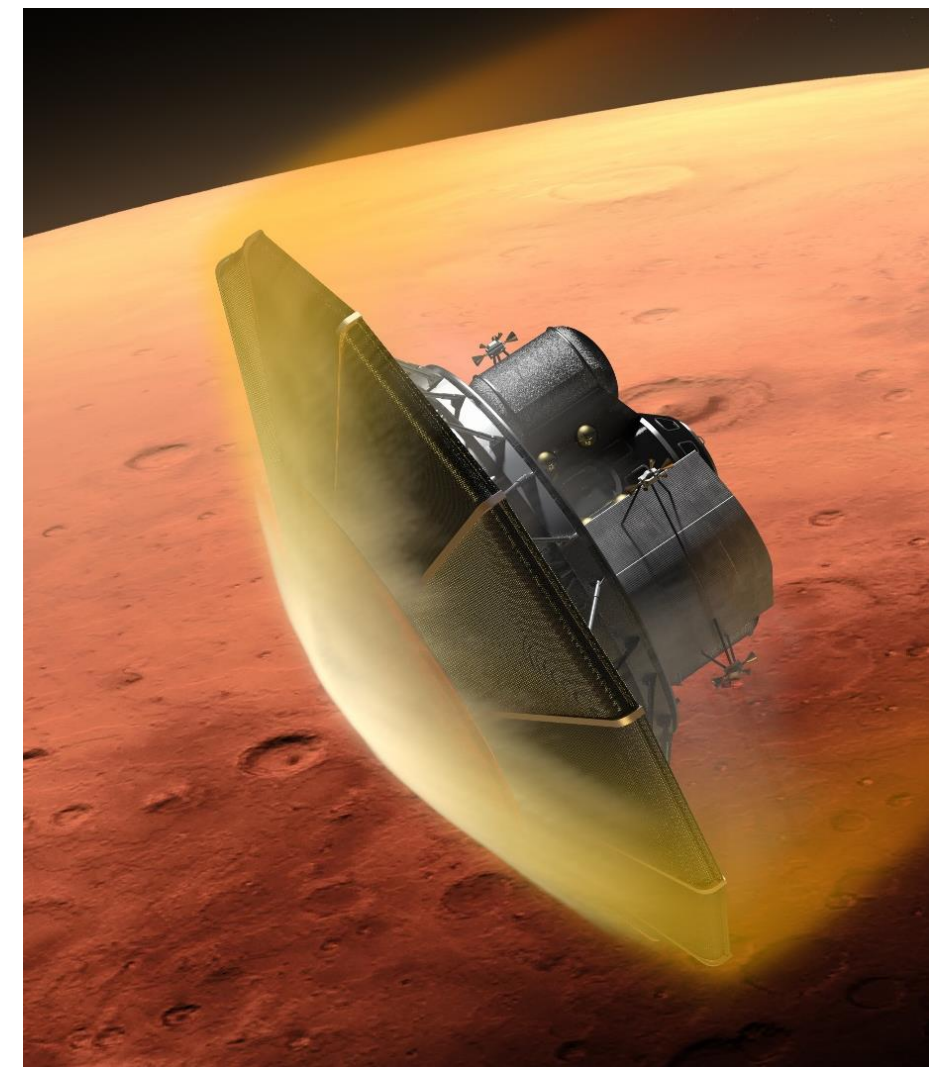

16m Lifting ADEPT Human Exploration 
NAgA 